\title{
Sexual dimorphism of hepatic gene expression: novel biological role of KRAB zinc finger repressors revealed
}

\author{
David J. Waxman ${ }^{1}$ and John L. Celenza \\ Division of Cell and Molecular Biology, Department of Biology, Boston University, Boston, Massachusetts 02215, USA
}

Significant physiological differences between the sexes exist, and underlying these differences are sex-dependent genetic regulatory events that are ultimately dictated by the sex chromosome genotype of the organism. For example, it has been known for many decades that the mammalian liver is a sexually dimorphic organ, exhibiting major sex differences in the profile of steroid and drug metabolism (Yates et al. 1958; Colby 1980). The physiological requirements for steroid hydroxylation differ between the sexes, and accordingly, many enzymes involved in steroid metabolism, most notably steroid hydroxylases belonging to the cytochrome P450 superfamily (Nelson 1999, 2003), are expressed in the liver in unique, sex-dependent patterns during postnatal and pubertal development (Waxman 1988; Shapiro et al. 1995). These sex differences are most dramatic in rodents, but may occur in humans as well, where they have an impact on cytochrome P450-catalyzed drug metabolism and pharmacokinetics (Jaffe et al. 2002; Meibohm et al. 2002). Early studies established that growth hormone $(\mathrm{GH})$, acting via its sex-dependent patterns of pituitary hormone secretion, is a major player in establishing and maintaining the sexual dimorphism of hepatic gene transcription that emerges in rodents at puberty (Gustafsson et al. 1983; Norstedt and Palmiter 1984). These patterns are ultimately determined by neonatal exposure to testosterone, which imprints on the hypothalamus the male program of neuroendocrine control of pulsatile pituitary GH secretion that is first seen at puberty and continues through adulthood (Jansson et al. 1985). The underlying molecular mechanisms that govern the sexdependent expression of hepatic cytochromes P450 and other enzymes, and their regulation by plasma $\mathrm{GH}$ profiles, are proposed to involve the stimulatory action of the signal transducer and transcriptional activator STAT5b, but are only partially understood (Waxman 2000; Schwartz 2001). Major new insight is provided by the discovery, reported in this issue of Genes \& Development, of a pair of KRAB (Krüppel-associated box) zinc

${ }^{1}$ Corresponding author.

E-MAIL djw@bu.edu; FAX (617) 353-7404.

Article and publication are at http://www.genesdev.org/cgi/doi/10.1101/ gad.1154603. finger genes, termed Rsl1 and Rs12, that can exert strong repression of male-specific hepatic gene expression in both males and females (Krebs et al. 2003). This repression is partially relieved in males at puberty in response to pulsatile GH signaling, greatly accentuating adult sex differences in gene expression.

\section{Rsl, a regulator of sex limitation}

The Rsl (Regulator of sex limitation) locus confers repression of sex-limited protein $(S I p)$, a gene that arose by duplication of the adjacent complement component C4 gene (Brown and Shreffler 1980). Slp is normally expressed only in adult male mice, where it responds to the positive effects of pulsatile GH stimulation (Georgatsou et al. 1993). Recessive rsl mutant alleles have arisen in populations of inbred mice and result in expression of Slp in female mice at $3 \mathrm{wk}$ of age (Tullis et al. 2003). Intriguingly, the increased expression of Slp observed in livers of intact rsl female mice is also seen in rsl male mice. Moreover, this effect is retained following hypophysectomy (Tullis et al. 2003). The action of Rsl is thus independent of the pituitary gland and its pulsatile release of $\mathrm{GH}$, which serves as the proximal inducer of Slp expression in intact male mice. Rsl likely serves as a repressor of $S I p$, as evidenced by the recessive nature of the $r s 1$ mutation and by the elevated expression in both female and male rsl mouse liver of Slp and several other male-specific, GH-regulated target genes (Tullis et al. 2003). These include Cyp2d9, a steroid hydroxylase cytochrome P450 gene (Sueyoshi et al. 1999), and genes belonging to the Mup (major urinary protein) family, which encodes male-specific pheromone-binding proteins (Johnson et al. 1995). However, efforts to elucidate the mechanism whereby Rsl acts would have to wait until the Rsl gene was identified. This has now been accomplished in an exciting study reported by Robins and coworkers in this issue of Genes \& Development (Krebs et al. 2003), where Rsl was identified as a repressor protein belonging to a large family of $\mathrm{KRAB}$ zinc finger genes for which no clearly defined in vivo functions had previously been identified. Most intriguing was the unanticipated finding that two Rsl genes are required for Rsl function. 


\section{Cloning of Rsl1 and Rsl2}

The Rsl locus was mapped previously to a 1.2 -cM region of mouse chromosome 13 (Jiang et al. 1996) and further reduced to a $1-\mathrm{Mb}$ DNA contig. Compounding the cloning effort undertaken by Krebs et al. (2003) was the presence in the 1-Mb Rs1 locus of 23 closely related genes encoding KRAB zinc finger proteins. Nonetheless, after refining the map position to an $\sim 200-\mathrm{kB}$ region containing eight $\mathrm{KRAB}$ zinc finger genes, two of the genes, initially termed Rslcan for Rsl candidate, were shown by Krebs et al. (2003) to harbor mutations in rsl mice. Rsl mice were found to contain intact Rslcan-9 (renamed Rsl1) and Rslcan-4 (renamed Rsl2) genes, whereas rsl mutant strains carry mutations in one, or both, genes: a mutation in Rslcan-9 disrupts splicing needed to generate a functional KRAB zinc finger protein, and a rearrangement of Rslcan-4 fuses Rslcan-4 to Rslcan-13, likely due to a homologous recombination-mediated deletion between these two adjacent genes.

Restoration of full wild-type Rsl function requires both Rsl1 and Rsl2, as Krebs et al. (2003) demonstrated by creating transgenic rsl mice carrying BAC clones containing either Rsl1 or Rsl2. These studies led to the unexpected discovery that Rs 11 and Rsl2 play distinct roles, with each gene apparently repressing a subset of malespecific liver genes: RsI1 represses expression of Slp and Cyp2d9, whereas Rs12 represses Mup gene expression. Neither Rsl affected the expression of the steroid $15 \alpha$ hydroxylase P450 gene Cyp2a4, which is expressed in a female-predominant pattern, suggesting that expression of female-predominant liver genes is unaffected by the Rsl locus.

\section{Rsl acting as a KRAB zinc finger repressor}

The discovery that Rs11 and Rs12 encode KRAB zinc finger proteins is consistent with earlier genetic analysis suggesting that $R s l$ acts by negative regulation and may function to repress transcription (Brown and Shreffler 1980; Tullis et al. 2003). KRAB zinc finger proteins, of which the mouse genome encodes over 100 members (Ravasi et al. 2003), are defined by a conserved N-terminal KRAB domain, a divergent linker region, and a Cterminal domain containing multiple Krüppel $\left(\mathrm{C}_{2} \mathrm{H}_{2}\right)$ type zinc finger motifs (Fig. 1A). The KRAB domain (Bellefroid et al. 1991), which appears only in vertebrate species (but not fish), contains a 42-amino acid KRAB A box and is usually followed by an $\sim 32$-amino acid KRAB B box (or a diverged KRAB b box; Looman et al. 2002; Shannon et al. 2003). All variants of the KRAB domain studied so far function by recruiting the transcriptional corepressor KAP1 (KRAB-associated protein-1) to DNA targets (Witzgall et al. 1994; Friedman et al. 1996; Kim et al. 1996; Moosmann et al. 1996). KAP1, also called TIF $\beta$ and KRIP-1, operates as a scaffold protein that recruits HP1 (heterochromatin protein 1), a chromatin organization modifier that serves as a dose-dependent regulator of heterochromatin-mediated gene silencing, as well as fac-

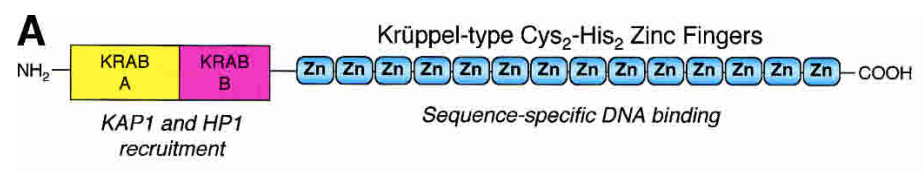

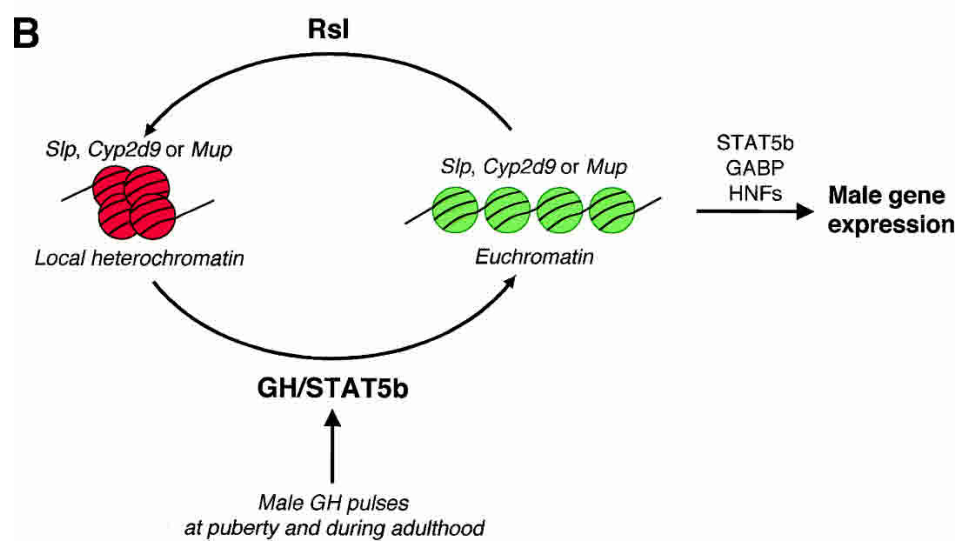

Figure 1. Model for Rsl regulation of sexually dimorphic liver gene expression. (A) Domain organization of the $\mathrm{KRAB}$ zinc finger proteins Rsll and Rsl2. The KRAB domain, consisting of an A box and a B box, recruits the corepressor KAP1 and the heterochromatin factor HP1, leading to localized formation of heterochromatin at target genes specified by the zinc finger domain. A total of $14 \mathrm{C}_{2} \mathrm{H}_{2}$-type zinc fingers, some degenerate, are found in Rsl proteins. (B) Model for the opposing actions of Rsl and $\mathrm{GH}$ pulse-activated STAT5b in regulating male-specific liver gene expression. Rsl is proposed to induce heterochromatin formation, characterized by localized formation of highly compact nucleosome arrays (red) and transcriptional silencing of the male-specific Slp, Cyp2d9, and Mup genes in both male and female mouse liver prior to puberty. This repression is selectively relieved at puberty in male mice through the action of pulsatile $\mathrm{GH}$, which is proposed to act via STAT5b and induce the conversion of heterochromatin to the more open and accessible euchromatin (green). Gene transcription can then be activated by positive regulatory factors, such as STAT5b, GABP, and various hepatocyte-enriched nuclear factors (HNFs). In the simplest model, Rsl binds directly to specific promoter elements upstream of its male-specific target genes, leading to recruitment of KAP1 and HP1 and inducing formation of a highly localized, stable form of heterochromatin, represented in this figure as four condensed nucleosomes. GH-activated STAT5b may negatively regulate Rsl repression by binding directly to STAT5 response elements in the vicinity of the HP1-condensed nucleosomes, followed by recruitment of factors required for nucleosome disassembly. Other GH-activated Rsl regulatory mechanisms are possible (see text). The diagrammatic representations of heterochromatin and euchromatin shown here are based on Dillon and Festenstein (2002). Other models for the nucleosomal structure of genes silenced by conversion to heterochromatin may also be considered, such as the site-exposure model of Ahmad and Henikoff (2002a). This latter model may help explain the requirement of high nuclear levels of STAT5b for male liver gene expression (Choi et al. 1999) and, presumably, for derepression of Rsl target genes, in terms of a requirement for a threshold concentration of STAT5b for binding to the unwrapped site on DNA. 
tors required for histone deacetylation and histone $\mathrm{H} 3$ Lys 9 methylation (Dillon and Festenstein 2002). Together, these events lead to the localized formation of highly compact nucleosome arrays and transcriptional silencing of target genes specified by the zinc finger domain. Variation in the number of zinc fingers and their amino acid sequences is thought to confer differences in DNA-binding specificity and hence differences in target gene recognition.

As outlined above, much is known about the biochemical properties of KRAB zinc finger proteins. However, given the unusually large number of $\mathrm{KRAB}$ zinc finger proteins encoded by both rodent and human genomes, it is surprising how little is known about their natural target genes, or in which regulatory pathways they might participate (Tanaka et al. 2002). Based on the expression patterns that characterize a number of KRAB zinc finger genes, and their responses to differentiation in various cell models, it has been speculated that KRABs play a role in differentiation and development (Peng et al. 2000). The study by Krebs et al. (2003) thus takes on added significance, as it is the first to provide a clear genetic assignment of function to a KRAB zinc finger gene.

As noted by Krebs et al. (2003), it will be important to determine whether S1p and other genes repressed in the female liver are direct targets of Rsl1 and Rs12, or if these Rsls act indirectly, that is, by repression of a strong transcriptional activator of the male-specific genes. In the simplest model, Rsl1 and Rsl2 interact directly, and in a differential manner, with the S1p, Cyp2d9 (Rsl1), and Mup gene promoters (Rsl2) to confer repression. The limited number of overall amino acid differences between Rsl1 and Rsl2 ( $6 \%$ divergence) is apparently sufficient for discrimination between DNA response elements or the associated transcriptional complexes that assemble on target genes. The KRAB A boxes of Rsl1 and Rsl2 differ by only 1 amino acid, whereas their KRAB B boxes differ by three residues. Given the role of the KRAB domain in KAP1 recruitment, noted above, these differences are unlikely to dictate Rsl target gene specificity. Rather, this function is likely to reside in the zinc finger domains. Rsl1 and Rsl2 each encode 14 zinc finger motifs, several of which are degenerate, with $94 \%$ overall amino acid sequence identity. These differences in sequence could account for different target selection or perhaps different affinities for the same targets by each Rsl. Another possibility is that promoter context, in combination with the relative abundance of each Rsl, determines target gene specificity (Krebs et al. 2003). Further complexity may be presumed based on an examination of the Rsl region of the mouse genome, which raises the possibility of complex patterns of splicing for Rsl1 and Rsl2. Unique splice variants generated from each locus could contribute to each Rsl's unique target gene specificity.

\section{Rsl-related genes-keeping it all in the family?}

In addition to $S 1 p, C y p 2 d 9$, and Mup, many other liverexpressed genes are known to be male-specific and GH- regulated, including various steroid sulfotransferases and other P450 steroid hydroxylases, and these could also be regulated by Rsl1 or Rsl2. Alternatively, other KRAB zinc finger proteins, perhaps closely related to Rsll and Rsl2, may contribute to the repression in female liver of these other genes. Rsl1 and Rsl2 are part of a short chromosome duplication, suggesting that they arose from a common ancestor capable of carrying out the entire RsI function. Although Rsl1 and RsI2 are most closely related to each other, they share a high degree of similarity with several adjacent KRAB zinc finger genes. These closely linked genes encode fairly well conserved KRAB A boxes and show greater divergence in the B box (Krebs et al. 2003). Although it is known that KRAB A boxes are sufficient to interact with KAP1 and cause repression (Witzgall et al. 1994; Kim et al. 1996), the role of the $\mathrm{KRAB} B$ box is less clear. The KRAB B box cannot repress gene expression on its own, but when combined with a $\mathrm{KRAB} A$ box the level of repression is increased significantly compared to the A box alone (Vissing et al. 1995). Thus, differences in the KRAB B box sequences between Rsl1, Rsl2, and neighboring KRAB zinc finger proteins (Krebs et al. 2003) may, as speculated in another context (Vissing et al. 1995), influence the degree of repression. Variation is also seen in the number and sequence of the zinc fingers encoded by $R s 11, R s 12$, and their neighboring genes, consistent with each protein repressing different gene targets. Nonetheless, overlap between targets is possible. For example, the $r_{s} l^{\mathrm{FM}}$ allele carries the same splice site mutation found in Rsl1 that is carried in the originally cloned $r s 1^{\mathrm{PL}}$ allele; however mutations in Rs12 are absent. Based on the complementation experiments reported by Krebs et al. (2003), in which a BAC containing wild-type $R S 11$ repressed Slp and Cyp2d9, and a BAC containing Rsl2 repressed Mup expression, $r s l^{\mathrm{FM}}$ mice should show no repression of Slp. However, these mice do show a significant amount of $S I p$ repression (Tullis et al. 2003), suggesting to Krebs et al. (2003) that Rsl2, or perhaps another related gene, can compensate for the absence of Rs11. In this regard, determination of the relative expression levels of Rsl1 and Rsl2 measured in RsI wild-type and mutant mice, combined with more quantitative data on target gene specificity, may help clarify how strict the division of labor is between the two genes.

\section{Derepression of gene silencing by male GH pulsation?}

In addition to its role in silencing male-specific genes in female liver tissue, $R s I$ can also repress the expression of Slp and other male-specific genes in male liver, albeit in an incomplete manner (Jiang et al. 1996; Tullis et al. 2003). Presumably, this incomplete repression reflects a balance between the intrinsic repressor activity of Rsl and the intrinsic stimulatory action of pulsatile GH signaling on male-specific gene transcription, which has been proposed to be mediated by STAT5b (Waxman et al. 1995; Waxman 2000). Mouse knockout studies have shown that the GH-responsive STAT5b plays a key role in regulating hepatic sexual dimorphism, with STAT5b deficiency leading to a selective loss of the expression of 
male-specific genes (Udy et al. 1997; Teglund et al. 1998). Accordingly, Rsl and STAT5b may play opposing roles, with Rsl repressing and STAT5b derepressing male-specific target genes (Fig. 1B).

The molecular details describing the opposing actions of Rsl and GH pulse signaling are wholly unknown, and are likely to be intriguing. Rsl could interact with STAT5b directly on the promoters of GH pulse-responsive, male-specific liver genes, which contain functional, albeit weak, STAT5b binding sites (Subramanian et al. 1998; Park and Waxman 2001). Alternatively, Rsl may block the action of one or more of the liver-enriched transcription factors (hepatocyte-enriched nuclear factors, HNFs) whose expression or activity is regulated by GH and STAT5b (Lahuna et al. 2000; Rastegar et al. 2000; Park and Waxman 2001; Piwien-Pilipuk et al. 2002) and may contribute to the sex-dependence of liver gene expression. Another possibility is that GH signaling negatively impacts Rsl at the protein level. Although the repression of SIp seen in both male and female Rsl mice evidences the expression of Rsl in both sexes, males and females may, nevertheless, exhibit important quantitative differences in Rsl protein levels or in Rsl posttranslational modification, either of which could be dictated by GH pulses and the STAT5b signaling pathway. The dramatic increase in expression of Slp seen at weaning $(3$ wk of age) in both rsl male and rsl female mice (Tullis et al. 2003) precedes by a full 2-3 wk the normal GH pulseinduced pubertal expression of SIp seen in Rsl males, and indicates that Rsl1, at least, is active during the pubertal period in both sexes.

Although GH and STAT5b signaling might antagonize Rsl function by one or more of the above mechanisms, a key question will be how these interactions lead to derepression of gene expression (Fig. 1B). Recent findings suggest that gene silencing mediated by KAP1 (and by association, KRAB zinc finger proteins) can be inherited in a mitotically stable manner (Ayyanathan et al. 2003), with KRAB zinc finger proteins targeting individual genes for silencing, leading to the localized formation of heterochromatin in otherwise euchromatic regions (discussed in Fahrner and Baylin 2003). The potential for temporal stability of KAP1-mediated repression, supported by these findings, suggests that Rsl1 or Rsl2 need not be expressed continuously throughout postnatal development to maintain the repressed state observed in female mice. Moreover, the model that Rsl-mediated repression is due to localized heterochromatin formation at target genes may help explain the requirement for several days of repeated GH pulsation to restore expression of male-specific liver P450 genes to GH-deficient males (Noshiro et al. 1986), even though STAT5b is maximally activated within 15 min of an initial plasma GH pulse (Waxman et al. 1995). KAP1-dependent methylation of histone H3 on Lys 9, necessary for heterochromatin formation, apparently cannot be reversed enzymatically in vivo; instead, nucleosomes containing Lys 9-methylated H3 must be replaced in order to convert back to active chromatin (Ahmad and Henikoff 2002a,b). Perhaps this slow conversion from heterochromatin to euchromatin accounts for the relatively slow induction of male-specific liver genes at puberty, extending over a period of days beginning at $\sim 5 \mathrm{wk}$ of age, and for the similar delay in gene re-activation by exogenous $\mathrm{GH}$ pulses in $\mathrm{GH}$ deficient mice or following hormone ablation by hypophysectomy. Further characterization of Rsl target genes and their chromatin structure under repressed and derepressed conditions will not only establish the mechanism by which Rsl represses gene expression, but may also shed light on how GH signaling interrupts Rsldependent gene silencing.

Additional support for Rsl-mediated heterochromatin formation being an important regulator of sexually dimorphic liver gene expression comes from earlier analysis of CpG methylation of the Slp and Cyp2d9 promoters. Hypermethylation of $\mathrm{CpG}$ correlates with repression of transcription and may play an active role in the formation and/or maintenance of heterochromatin (Tate and Bird 1993; Cameron et al. 1999). A CpG site within a proximal promoter element conserved in Slp and Cyp2d9 is hypermethylated in female compared to male liver (Yokomori et al. 1995a,b), supporting the hypothesis that in female livers the SIp and Cyp2d9 promoters are in a more highly condensed chromatin structure than in males. Demethylation of this CpG site during postnatal male development (Yokomori et al. 1995a) correlates with the pubertal induction of plasma GH pulsation, liver STAT5b activation, and male-specific gene expression (Choi and Waxman 2000). Furthermore, GA-binding protein (GABP), a methylation-sensitive Ets-related transcription factor, preferentially binds to the demethylated $\mathrm{CpG}$ sequence found in males and trans-activates the Cyp2d9 promoter (Yokomori et al. 1995a). These observations suggest the following model as a working hypothesis (Fig. 1B), whereby male-specific liver gene expression is induced at puberty: Male plasma GH pulses, acting in a STAT5b-dependent manner, reverse Rsl-mediated gene silencing by inducing nucleosome replacement and CpG demethylation at the Cyp2d9 and $S 1 p$ promoters to locally convert heterochromatin to euchromatin. This, in turn, enhances the binding of established trans-activating factors, such as STAT5b, GABP, and various liver-enriched transcription factors, several of which are regulated in a $\mathrm{GH}$-dependent manner, as noted above. Finally, because the conversion of heterochromatin to euchromatin seems likely to be an all-or-nothing event for any single individual gene in a given cell, the partial repressor function of Rsl that is manifest in adult male liver in vivo (Jiang et al. 1996; Tullis et al. 2003) may reflect transcriptional silencing of the male-specific genes in a subpopulation of liver cells (e.g., periportal or perivenous hepatocytes), rather than reduced expression of Slp and the other Rsl target genes in 100\% of the cells.

The stable, heritable nature of gene silencing induced by KAP1 tethered to KRAB zinc finger proteins (Ayyanathan et al. 2003) may make Rsl proteins ideally suited for achieving the strong repression of male-specific gene expression that persists in prepubertal rodent liver, and for enforcing the male specificity of gene expression that results from Rsl action in female liver through adult- 
hood. Rsl and KAP1 may thus serve as effective corepressors of GH action that respond slowly to physiological changes in hormonal status, including situations that require stable changes in the developmental profile of gene expression (e.g., at puberty). As such, this mechanism may differ in one or more fundamental ways from that employed by another class of corepressors of hormone action, namely, the non-DNA-binding nuclear receptor corepressors, which actively repress gene expression in the absence of classic steroid/nuclear receptor agonists and respond rapidly to hormonal stimulation (Jones and Shi 2003).

\section{Rsl: the cheese does not stand alone}

STAT5b deficiency leads to the up-regulation in male mouse liver of several GH-regulated P450 genes whose expression is normally restricted to adult females (Udy et al. 1997; Park et al. 1999). Accordingly, STAT5b may activate (or induce) in male liver a repressor of femalespecific genes that serves as a counterpart to the malespecific gene repressor function that Rsl exhibits in females. Indeed, the female-specific mouse steroid hydroxylase $\mathrm{P} 450$ gene Cyp2a4 shows GH pulse-dependent repression in males that is mediated by a trans-acting locus termed Gdr (GH-dependent repression; Aida and Negishi 1993). A mouse strain that carries a recessive mutation in $G d r$ (BALB/cJ mice) shows a loss of this repression in males. Conceivably, $G d r$ may encode a $\mathrm{KRAB}$ zinc finger protein that represses the femalespecific P450 gene Cyp2a4 in male mouse liver in a way that is analogous to the repression that Rsl confers to malespecific genes in female liver. It will be interesting to test this hypothesis and to determine whether male GH pulses, acting via STAT5b, use the same mechanism to activate Gdr, the repressor of Cyp2a4, as is used to inactivate Rsl, the repressor of Slp, Cyp2d9, and Mup.

Clearly, the genetic regulation of sexually dimorphic liver gene expression in mice is complicated and not controlled by a single mechanism. Although GH signaling, mediated by STAT5b, and Rsl-dependent euchromatin to heterochromatin interconversion appear to be the two major players in the liver, these factors have limited roles in regulation of sexually dimorphic gene expression in other tissues. Several genes that display male-specific GH-regulated expression, including genes shown to be Rsl target genes, also display male-specific expression in kidney (Sundseth and Waxman 1992; Nelson and Robins 1997). In kidney, however, testosterone and not GH serves as the proximal inducer of gene expression. Correspondingly, the Rsl locus has no impact on the sex specificity of gene expression in the kidney (Tullis et al. 2003).

Finally, it is apparent from comparisons of laboratory and "wild" mouse strains that there is variation in both the array of genes expressed in a sexually dimorphic manner and in the magnitude of the differences in expression between the sexes. Complex environmental selections are likely at work for and against expression of these genes. All rsl mice examined carry the same mutant rsl1 allele (Krebs et al. 2003), suggesting a single occurrence for this mutation in the mouse population. rsl2 might have been additionally selected in $r s 1^{\mathrm{PL}}$ strains to enhance the rsl phenotype which, as suggested by Krebs et al. (2003), would lead to earlier litters, a selective advantage in the laboratory. However, loss of sexually dimorphic expression, at least for some genes, also occurs in "wild" mice (Sueyoshi et al. 1995), where it may be advantageous for females to lose dimorphic expression and acquire the ability to express certain genes, for example, male-specific P450s that in certain environments may confer a selective advantage in terms of added capacity for foreign compound metabolism.

\section{Rsl: a rodent-specific phenomenon?}

Although sexually dimorphic liver function, including sexually dimorphic P450-catalyzed drug metabolism, is seen in humans, it is unclear whether Rsl function is conserved between rodents and humans. As noted by Krebs et al. (2003), the region of mouse chromosome 13 that contains the Rsl locus is not syntenic with the human genome. In addition, although the KRAB zinc finger gene family is likely quite extensive in humans, comprised of at least 100 members (Looman et al. 2002), orthologs of Rsl1 and Rsl2, as determined by sequence comparison, may not exist. Furthermore, as Krebs et al. (2003) noted, the known targets of Rsl belong to multigene families that lack readily identifiable orthologs in humans and may contribute to speciation, in particular as it relates to reproduction. Indeed, many of the sexdependent, plasma GH profile-regulated proteins found in mouse and rat liver are enzymes that catalyze speciesspecific patterns of steroid metabolism (P450 steroid hydroxylases, sulfotransferases, etc.) or liver secretory proteins, such as MUPs, which regulate species-specific aspects of reproduction, including sexual maturation, estrus synchronization, and male exploratory behavior in female mice (Novotny et al. 1999). Distinct mechanisms for regulation of sexually dimorphic gene expression may therefore have arisen in humans compared to mice. Nonetheless, if KRAB zinc finger proteins play a major role in dynamically regulating gene expression in mammals, they will undoubtedly be used to control key aspects of human biology such as sexual development.

The cloning and identification of $R s l$ genes in the accompanying article by Krebs et al. (2003), combined with the existence of $r s 1$ mutant mice and the rich history of physiological and metabolic studies on the impact of liver sexual dimorphism in rodents, provide an important biological context to study KRAB zinc finger protein function. Future studies will not only shed light on the regulation of sexually dimorphic gene expression, but the Rsl system will serve as a much needed model for understanding the role of KRAB zinc finger proteins in targeted repression of gene expression.

\section{Acknowledgments}

This work was supported in part by NIH grant DK33765 (D.J.W.) and USDA grant 2002-35318-12715 (J.L.C.). We apologize to colleagues whose work was not cited due to space constraints. 


\section{References}

Ahmad, K. and Henikoff, S. 2002a. Epigenetic consequences of nucleosome dynamics. Cell 111: 281-284.

. 2002b. The histone variant H3.3 marks active chromatin by replication-independent nucleosome assembly. Mol. Cell 9: 1191-1200.

Aida, K. and Negishi, M. 1993. A trans-acting locus regulates transcriptional repression of the female-specific steroid 15 $\alpha$-hydroxylase gene in male mice. J. Mol. Endocrinol. 11: 213-222.

Ayyanathan, K., Lechner, M.S., Bell, P., Maul, G.G., Schultz, D.C., Yamada, Y., Tanaka, K., Torigoe, K., and Rauscher III, F.J. 2003. Regulated recruitment of HP1 to a euchromatic gene induces mitotically heritable, epigenetic gene silencing: A mammalian cell culture model of gene variegation. Genes \& Dev. 17: 1855-1869.

Bellefroid, E.J., Poncelet, D.A., Lecocq, P.J., Revelant, O., and Martial, J.A. 1991. The evolutionarily conserved Kruppelassociated box domain defines a subfamily of eukaryotic multifingered proteins. Proc. Nat1. Acad. Sci. 88: 3608-3612.

Brown, L.J. and Shreffler, D.C. 1980. Female expression of the $\mathrm{H}$-2-linked sex-limited protein (Slp) due to non-H-2 genes. Immunogenetics 10: 19-29.

Cameron, E.E., Bachman, K.E., Myohanen, S., Herman, J.G., and Baylin, S.B. 1999. Synergy of demethylation and histone deacetylase inhibition in the re-expression of genes silenced in cancer. Nat. Genet. 21: 103-107.

Choi, H.K. and Waxman, D.J. 1999. Growth hormone, but not prolactin, maintains, low-level activation of STAT5a and STAT5b in female rat liver. Endocrinology 140: 5126-5135.

- 2000. Plasma growth hormone pulse activation of hepatic JAK-STAT5 signaling: Developmental regulation and role in male-specific liver gene expression. Endocrinology 141: 3245-3255.

Colby, H.D. 1980. Regulation of hepatic drug and steroid metabolism by androgens and estrogens. Adv. Sex Horm. Res. 4: 27-71.

Dillon, N. and Festenstein, R. 2002. Unravelling heterochromatin: Competition between positive and negative factors regulates accessibility. Trends Genet. 18: 252-258.

Fahrner, J.A. and Baylin, S.B. 2003. Heterochromatin: Stable and unstable invasions at home and abroad. Genes \& Dev. 17: 1805-1812.

Friedman, J.R., Fredericks, W.J., Jensen, D.E., Speicher, D.W., Huang, X.P., Neilson, E.G., and Rauscher III, F.J. 1996. KAP1 , a novel corepressor for the highly conserved KRAB repression domain. Genes \& Dev. 10: 2067-2078.

Georgatsou, E., Bourgarel, P., and Meo, T. 1993. Male-specific expression of mouse sex-limited protein requires growth hormone, not testosterone. Proc. Nat1. Acad. Sci. 90: 36263630 .

Gustafsson, J.A., Mode, A., Norstedt, G., and Skett, P. 1983. Sex steroid induced changes in hepatic enzymes. Annu. Rev. Physiol. 45: 51-60.

Jaffe, C.A., Turgeon, D.K., Lown, K., Demott-Friberg, R., and Watkins, P.B. 2002. Growth hormone secretion pattern is an independent regulator of growth hormone actions in humans. Am. J. Physiol. Endocrinol. Metab. 283: 1008-1015.

Jansson, J.O., Eden, S., and Isaksson, O. 1985. Sexual dimorphism in the control of growth hormone secretion. Endocr. Rev. 6: $128-150$.

Jiang, P.P., Frederick, K., Hansen, T.H., and Miller, R.D. 1996. Localization of the mouse gene releasing sex-limited expression of Slp. Proc. Nat1. Acad. Sci. 93: 913-917.

Johnson, D., al-Shawi, R., and Bishop, J.O. 1995. Sexual dimor- phism and growth hormone induction of murine pheromone-binding proteins. J. Mol. Endocrinol. 14: 21-34.

Jones, P.L. and Shi, Y.B. 2003. N-CoR-HDAC corepressor complexes: Roles in transcriptional regulation by nuclear hormone receptors. Curr. Top. Microbiol. Immunol. 274: 237268.

Kim, S.S., Chen, Y.M., Oleary, E., Witzgall, R., Vidal, M., and Bonventre, J.V. 1996. A novel member of the RING finger family, KRIP-1, associates with the KRAB-A transcriptional repressor domain of zinc finger proteins. Proc. Natl. Acad. Sci. 93: 15299-15304.

Krebs, C.J., Larkins, L.K., Price, R., Tullis, K.M., Miller, R.D., and Robins, D.M. 2003. Regulator of sex-limitation (RsI) encodes a pair of KRAB zinc-finger genes that control sexually dimorphic liver gene expression. Genes \& Dev. (this issue).

Lahuna, O., Rastegar, M., Maiter, D., Thissen, J.P., Lemaigre, F.P., and Rousseau, G.G. 2000. Involvement of STAT5 (signal transducer and activator of transcription 5) and HNF-4 (hepatocyte nuclear factor 4) in the transcriptional control of the hnf6 gene by growth hormone. Mol. Endocrinol. 14: 285 294.

Looman, C., Abrink, M., Mark, C., and Hellman, L. 2002. KRAB zinc finger proteins: An analysis of the molecular mechanisms governing their increase in numbers and complexity during evolution. Mol. Biol. Evol. 19: 2118-2130.

Meibohm, B., Beierle, I., and Derendorf, H. 2002. How important are gender differences in pharmacokinetics? Clin. Pharmacokinet. 41: 329-342.

Moosmann, P., Georgiev, O., Le Douarin, B., Bourquin, J.P., and Schaffner, W. 1996. Transcriptional repression by RING finger protein TIF1 $\beta$ that interacts with the KRAB repressor domain of KOX1. Nucleic Acids Res. 24: 4859-4867.

Nelson, D.R. 1999. Cytochrome P450 and the individuality of species. Arch. Biochem. Biophys. 369: 1-10.

- 2003. Comparison of P450s from human and fugu: 420 million years of vertebrate P450 evolution. Arch. Biochem. Biophys. 409: 18-24.

Nelson, S.A. and Robins, D.M. 1997. Two distinct mechanisms elicit androgen-dependent expression of the mouse sex-limited protein gene. Mol. Endocrinol. 11: 460-469.

Norstedt, G. and Palmiter, R. 1984. Secretory rhythm of growth hormone regulates sexual differentiation of mouse liver. Cell 36: $805-812$.

Noshiro, M., Serabjit-Singh, C.J., Bend, J.R., and Negishi, M. 1986. Female-predominant expression of testosterone 16 $\alpha$-hydroxylase ("I" $-\mathrm{P}-450(16) \alpha)$ and its repression in strain 129/J. Arch. Biochem. Biophys. 244: 857-864.

Novotny, M.V., Ma, W., Wiesler, D., and Zidek, L. 1999. Positive identification of the puberty-accelerating pheromone of the house mouse: The volatile ligands associating with the major urinary protein. Proc. $R$ Soc. Lond. B Biol. Sci. 266: 2017-2022.

Park, S.H. and Waxman, D.J. 2001. Inhibitory cross-talk between STAT5b and liver nuclear factor HNF3 $\beta$ : Impact on the regulation of growth hormone pulse-stimulated, malespecific liver cytochrome P-450 gene expression. I. Biol. Chem. 276: 43031-43039.

Park, S.H., Liu, X., Hennighausen, L., Davey, H.W., and Waxman, D.J. 1999. Distinctive roles of STAT5a and STAT5b in sexual dimorphism of hepatic P450 gene expression. Impact of STAT5a gene disruption. J. Biol. Chem. 274: 7421-7430.

Peng, H., Begg, G.E., Harper, S.L., Friedman, J.R., Speicher, D.W., and Rauscher III, F.J. 2000. Biochemical analysis of the Kruppel-associated box (KRAB) transcriptional repression domain. J. Biol. Chem. 275: 18000-18010. 
Piwien-Pilipuk, G., MacDougald, O., and Schwartz, J. 2002. Dual regulation of phosphorylation and dephosphorylation of C/EBP $\beta$ modulate its transcriptional activation and DNA binding in response to growth hormone. I. Biol. Chem. 277: 44557-44565.

Rastegar, M., Lemaigre, F.P., and Rousseau, G.G. 2000. Control of gene expression by growth hormone in liver: Key role of a network of transcription factors. Mol. Cell Endocrinol. 164: $1-4$.

Ravasi, T., Huber, T., Zavolan, M., Forrest, A., Gaasterland, T., Grimmond, S., and Hume, D.A. 2003. Systematic characterization of the zinc-finger-containing proteins in the mouse transcriptome. Genome Res. 13: 1430-1442.

Schwartz, J. 2001. Editorial: Pulsatile hormone patterns governing transcription factor function. Physiology of episodic GH secretion. Endocrinology 142: 4595-4598.

Shannon, M., Hamilton, A.T., Gordon, L., Branscomb, E., and Stubbs, L. 2003. Differential expansion of zinc-finger transcription factor loci in homologous human and mouse gene clusters. Genome Res. 13: 1097-1110.

Shapiro, B.H., Agrawal, A.K., and Pampori, N.A. 1995. Gender differences in drug metabolism regulated by growth hormone. Int. J. Biochem. Cell Biol. 27: 9-20.

Subramanian, A., Wang, J., and Gil, G. 1998. STAT 5 and NF-Y are involved in expression and growth hormone-mediated sexually dimorphic regulation of cytochrome P450 3A10/ lithocholic acid 6ß-hydroxylase. Nucleic Acids Res. 26: $2173-2178$.

Sueyoshi, T., Kobayashi, R., Nishio, K., Aida, K., Moore, R., Wada, T., Handa, H., and Negishi, M. 1995. A nuclear factor (NF2d9) that binds to the male-specific P450 (Cyp 2d-9) gene in mouse liver. Mol. Cell. Biol. 15: 4158-4166.

Sueyoshi, T., Yokomori, N., Korach, K.S., and Negishi, M. 1999. Developmental action of estrogen receptor- $\alpha$ feminizes the growth hormone-Stat5b pathway and expression of Cyp2a4 and Cyp2d9 genes in mouse liver. Mol. Pharmacol. 56: 473477.

Sundseth, S.S. and Waxman, D.J. 1992. Sex-dependent expression and clofibrate inducibility of cytochrome P450 4A fatty acid $\omega$-hydroxylases. Male specificity of liver and kidney CYP4A2 mRNA and tissue-specific regulation by growth hormone and testosterone. J. Biol. Chem. 267: 3915-3921.

Tanaka, K., Tsumaki, N., Kozak, C.A., Matsumoto, Y., Nakatani, F., Iwamoto, Y., and Yamada, Y. 2002. A Kruppel-associated box-zinc finger protein, NT2, represses cell-type-specific promoter activity of the $\alpha$ 2(XI) collagen gene. Mol. Cell. Biol. 22: 4256-4267.

Tate, P.H. and Bird, A.P. 1993. Effects of DNA methylation on DNA-binding proteins and gene expression. Curr. Opin. Genet. Dev. 3: 226-231.

Teglund, S., McKay, C., Schuetz, E., van Deursen, J.M., Stravopodis, D., Wang, D., Brown, M., Bodner, S., Grosveld, G., and Ihle, J.N. 1998. Stat5a and Stat5b proteins have essential and nonessential, or redundant, roles in cytokine responses. Cell 93: $841-850$.

Tullis, K.M., Krebs, C.J., Leung, J.Y., and Robins, D.M. 2003. The regulator of sex-limitation gene, rsl, enforces male-specific liver gene expression by negative regulation. Endocrinology 144: 1854-1860.

Udy, G.B., Towers, R.P., Snell, R.G., Wilkins, R.J., Park, S.H., Ram, P.A., Waxman, D.J., and Davey, H.W. 1997. Requirement of STAT5b for sexual dimorphism of body growth rates and liver gene expression. Proc. Natl. Acad. Sci. 94: 72397244.

Vissing, H., Meyer, W.K.H., Aagaard, L., Tommerup, N., and
Thiesen, H.J. 1995. Repression of transcriptional activity by heterologous krab domains present in zinc-finger proteins. FEBS Lett. 369: 153-157.

Waxman, D.J. 1988. Interactions of hepatic cytochromes P-450 with steroid hormones. Regioselectivity and stereospecificity of steroid metabolism and hormonal regulation of rat P-450 enzyme expression. Biochem. Pharmacol. 37: 71-84.

- 2000. Growth hormone pulse-activated STAT5 signalling: A unique regulatory mechanism governing sexual dimorphism of liver gene expression. Novartis Found. Symp. 227: 61-74; discussion 75-81.

Waxman, D.J., Ram, P.A., Park, S.H., and Choi, H.K. 1995. Intermittent plasma growth hormone triggers tyrosine phosphorylation and nuclear translocation of a liver-expressed, Stat 5-related DNA binding protein. Proposed role as an intracellular regulator of male-specific liver gene transcription. J. Biol. Chem. 270: 13262-13270.

Witzgall, R., O'Leary, E., Leaf, A., Onaldi, D., and Bonventre, J.V. 1994. The Kruppel-associated box-A (KRAB-A) domain of zinc finger proteins mediates transcriptional repression. Proc. Nat1. Acad. Sci. 91: 4514-4518.

Yates, F.E., Herbst, A.L., and Urquhart, J. 1958. Sex difference in rate of ring A reduction of $\delta 4-3$-keto-steroids in vitro by rat liver. Endocrinology 63: 887-902.

Yokomori, N., Kobayashi, R., Moore, R., Sueyoshi, T., and Negishi, M. 1995a. A DNA methylation site in the male-specific P450 (Cyp 2d-9) promoter and binding of the heteromeric transcription factor GABP. Mol. Cell. Biol. 15: 5355-5362.

Yokomori, N., Moore, R., and Negishi, M. 1995b. Sexually dimorphic DNA demethylation in the promoter of the Slp (sex-limited protein) gene in mouse liver. Proc. Natl. Acad. Sci. 92: 1302-1306. 


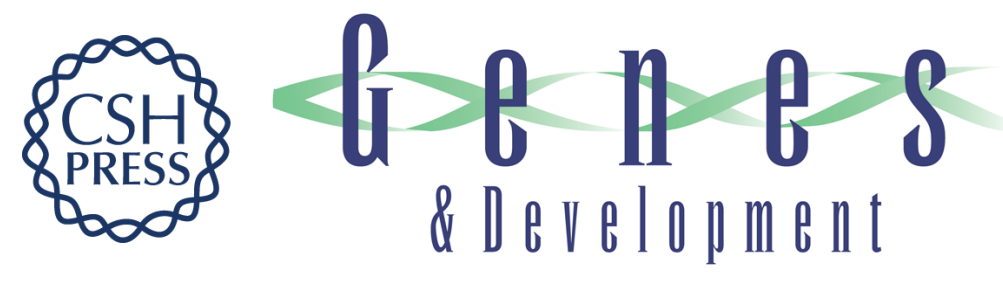

\section{Sexual dimorphism of hepatic gene expression: novel biological role of KRAB zinc finger repressors revealed}

David J. Waxman and John L. Celenza

Genes Dev. 2003, 17:

Access the most recent version at doi:10.1101/gad.1154603

References This article cites 56 articles, 24 of which can be accessed free at: http://genesdev.cshlp.org/content/17/21/2607.full.html\#ref-list-1

License

Email Alerting

Receive free email alerts when new articles cite this article - sign up in the box at the top Service right corner of the article or click here.

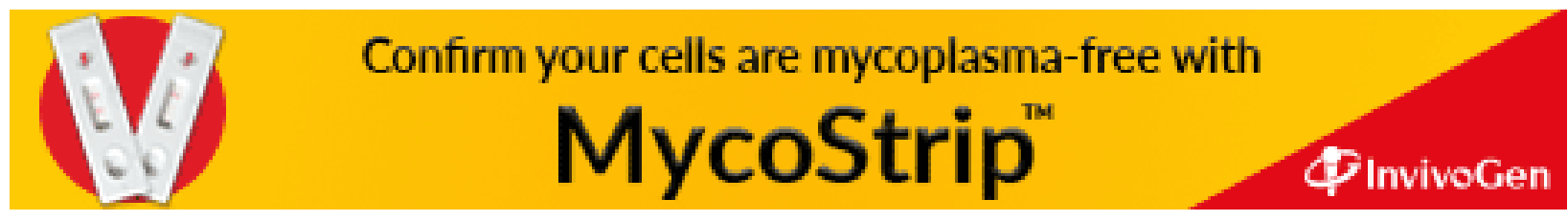

